\title{
Guidelines for Immersive Virtual Reality in Psychological Research
}

\author{
Madis Vasser ${ }^{1}$, Jaan Aru $^{1,2}$ \\ ${ }^{1}$ Institute of Computer Science, University of Tartu, Estonia \\ ${ }^{2}$ Institute of Biology, Humboldt University Berlin, Germany \\ E-mail address: Madis.vasser@ut.ee; Jaan.aru@gmail.com (corresponding author) \\ Full postal address: Institute of Computer Science, University of Tartu, Narva mnt 18, 51009 \\ Tartu, Estonia
}

\begin{abstract}
Virtual reality (VR) holds immense promise as a research tool to deliver results that are generalizable to the real world. However, the methodology used in different VR studies varies substantially. While many of these approaches claim to use "immersive VR", the different hardware and software choices lead to issues regarding reliability and validity of psychological VR research. Questions arise about quantifying presence, the optimal level of graphical realism, the problem of being in dual-realities and reproducibility of VR research. We discuss how VR research paradigms could be evaluated and offer a list of practical recommendations to have common guidelines for psychological VR research.
\end{abstract}

Published in Current Opinion in Psychology: https://doi.org/10.1016/j.copsyc.2020.04.010

\section{Introduction}

The number of annually published articles in peer reviewed academic journals with "virtual reality" as a keyword has doubled between 2016 and 2019 from around 6000 entries to over 12 000, according to EBSCO Information Services. The excitement is understandable as VR holds a huge potential for both basic science $\left[1^{* *}, 2^{* *}, 3\right]$ as well as therapeutic approaches $\left[4^{*}\right]$.

While the field of contemporary VR research emerged already in the early 90s, the recent surge in publications is associated with the release of several affordable and high-quality VR head-mounted displays (HMDs) $\left[1^{* *}, 5\right]$. Off-the-shelf consumer-oriented systems like the HTC Vive and Oculus Rift have given more researchers the opportunity to pursue psychological experiments with immersive VR setups - that is, integrating computers, HMDs, body-tracking sensors, special interface devices and 3D graphics [4*]. Given recent advancements in computational power, software and optics, these "new generation" devices offer low latency, wide field of view and unprecedented level of both interactivity and data gathering $\left[6^{* *}, 7,8^{*}\right]$, differing clearly from "non-immersive", "desktop" or projection-based VR solutions. However, 
there still exists a large heterogeneity among various contemporary approaches. We argue that considerations regarding hardware capabilities and software limitations should be taken into account when conducting and reporting psychological VR studies to resolve the situation that some experts have described as the "Wild West" with a "lack of clear guidelines and standards." $\left[9^{* *}\right]$.

\section{Ensuring Reliability}

The ultimate aim of a VR system is to technologically immerse the user into virtual worlds, inducing a sense of presence - the illusion of being in the virtual world and behaving accordingly [5]. A basic modern VR lab is relatively easy and affordable to set up with minimal prior knowledge $\left[1^{* *}, 6^{* *}\right]$. However, not all immersive VR systems provide the same level of presence [10]. This is a problem, as two studies, both using "immersive VR" might arrive at different results because the setups provided a different level of presence. Unfortunately, presence is hard to quantify. State of the art methods for measuring presence are mainly subjective self-report questionnaires and many popular scales developed before the new generation VR era are incapable of assessing qualitative differences between various modern immersive techniques $\left[11^{* *}\right]$.

An immersive VR setup can physically have many different configurations in various labs, some likely resulting in sub-optimal performance of the VR system, as technological knowledge of VR is not yet widespread in experimental science $\left[6^{* *}\right]$. The current immersive VR "gold standard" experience implements a room-scale solution, where the user can roam freely in a given play area of many square meters $\left[6^{* *}, 12,13\right]$. This allows for actual walking and exploration, crouching and other large movements. Such devices with consumer-optimized positional tracking solutions can introduce a possible interaction between the particular physical lab space and digital experience - while the user moves around in the virtual space, there might be specific moment in the study (e.g. user covering the tracking sensor while performing some motion) or problematic spots in the room where positional tracking is not guaranteed [14]. As a solution, experimenters should test each paradigm exhaustively and also be aware and design around the possible hardware limitations [15]. Information regarding the exact setup of the physical space should also be mentioned in the methods section of published papers. This would also answer calls to enhance reproducibility and transparency of VR research [16**].

Content wise, questions about reliability arise regarding the level of realism one should strive for in development. This could pose potential reliability problems when comparing two immersive VR methods that claim to study the same phenomenon, yet fail to attain a similar level of graphical fidelity $\left[15,17^{*}\right]$, for example if one study does not provide shadows and realistic colouring of objects. The advent of powerful graphical processing units and game engines have certainly made achieving a high graphical fidelity easier than ever [18*]. Although perceptually convincing VR scenarios are becoming the norm in VR development, it is still debated whether the effort to increase visual realism actually improves VR experiences and study outcomes 
$\left[1^{* *}, 4^{*}, 19^{* *}\right]$. However, we think that most studies comparing the effect of different graphical quality levels suffer from a floor effect due to limited technological capabilities in the past. Some types of studies may even benefit from purposefully "impoverished" visuals $\left[17^{*}, 20\right]$. Realistic graphics may certainly raise face validity and user buy-in of virtual experiences [4*]. Higher resemblance of reality can make the participants feel more present in the VR world [21*], with simulations high in context-realism and perspectival-fidelity producing "virtually-real experiences" $\left[1^{*}\right]$. Still, many scientific VR experiments even today are visually sub-standard when compared to contemporary content created for entertainment purposes that participants have become well accustomed to in their daily lives. Rendering virtual humans should not be semi-realistic, as this can induce the unwanted "uncanny valley" effect, whereby quasi-human characters can lead to revulsion [18*]. Many studies compare real-life situations with "identical" virtual copies, meticulously recreating physical testing laboratories in VR $\left[22^{*}, 23\right]$. However, digital copies often lack the most basic crucial properties of our everyday experience, such as object shadows and the user's correct head height. Some researchers, admitting shortcomings in the technical abilities of many academic developers, have proposed using commercially available games with high production value for research [24] and borrowing game design concepts from the entertainment sector [25]. More enjoyable content could also help draw in more study participants.

\section{Validity pitfalls}

Immersive VR research can potentially study and unravel processes that are closer to reality than classic laboratory experiments $\left[1^{* *}, 2^{* *}\right]$. This is important as generalizability to everyday life is critical for psychology as a science [ $\left.2^{\star *}\right]$. However, ecological validity of behavioural studies in VR is not a given even when immersive VR is used [26].

One prominent feature of VR that should not be ignored in psychological research is that of being in "dual realities" [27]. It is unclear whether VR engages the same cognitive processes as the real world, if the participant has top-down knowledge that the virtual world is not a substitution of the real world, but an add-on. The act of donning a HMD to enter the virtual world has been referred to as the device-gap, providing a clear and present demarcation between reality and VR [19**]. Some researchers have proposed to measure the persistent duality by counting self-reported "breaks in presence" [28] or drawing presence fluctuations post-experience [29*]. A theoretical exception to this problem would be perfect simulation of all possible senses, where the user truly becomes unaware of the base reality [30], a state called superrealism [19**].

Another problem is that different types of users get immersed differently [31] and the feeling of presence in a virtual environment relies heavily on suspension of disbelief, which is difficult to control [26] and may be a trait rather than a state [32]. Individual differences could be caused by differences in prior experience of VR $\left[1^{* *}\right]$. As a general rule of thumb, people with excessive 
VR experience should not be recruited as participants. Care should be taken to avoid gender bias towards male participants, a problem present in current VR research [33].

Specific components of the virtual experience can influence the phenomenon under investigation. For example, the presence of a realistic and believable virtual body can be critical to many studies $\left[1^{* \star}, 34^{* *}\right]$. To minimize possible confounds, standardized, validated and VR optimized libraries of 3D objects have become available [35,36]. However, due to fewer binocular cues and conflicting depth information inherent in current VR display technologies, it is questionable whether objects in VR elicit fine-tuned neural and behavioural responses representative of real objects $\left[34^{\star *}\right]$.

Many VR studies implement artificial movement for example by smooth virtual locomotion while the user remains physically stationary, which can be a source of cybersickness $\left[6^{* *}, 13,37\right]$. Designing around this pitfall should be kept in mind, as cybersickness substantially decreases cognitive performance and confounds physiological data [6** . However, given the recent advancements of developer knowledge, cybersickness can be largely avoided without decreasing the level of presence $\left[1^{* *}, 6^{* *}, 13,37,38\right]$

Finally, effects established in a classical highly controlled non-VR environment could disappear in a more complex virtual setting [25]. This direct comparison between VR and classical setups can be enabled in two ways - some researchers have conducted laboratory-like experiments in an endless uniform gray VR void [39], while others have gone to great lengths in reproducing the exact laboratory environment digitally [22*].

While VR gives experimenters the power to create faithful copies of reality, it also allows researchers to modify and break the basic laws governing our world in order to gain new insights into the workings of the human mind. Recent studies have flipped visual feedback to study visuomotor adaptation and sensory recalibration [40], modified gravity to assess physical judgments [41], turned participants hands invisible to study sensory attenuation of self-generated movements [39,42], observed non-Euclidean navigation [43] and allowed participants to embody elongated arms and virtual cardboard boxes [44]. We have no doubt that this represents just the beginning of the many possibilities offered by "Virtual Unreality".

\section{Evaluating VR paradigms}

As the consumer VR content market has expanded greatly in recent years, some basic "comfort rating" systems have emerged ${ }^{1}$. However, these simple criteria might be inadequate to capture

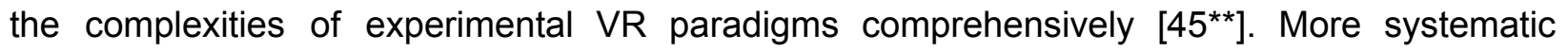
evaluation approaches have recently been proposed.

\footnotetext{
${ }^{1}$ https://support.oculus.com/comfort/ (accessed on 25.04.2020)
} 
The VR-Check framework $\left[45^{\star *}\right]$ evaluates ten main dimensions: cognitive domain specificity, ecological relevance, technical feasibility, user feasibility, user motivation, task adaptability, performance quantification, immersive capacities, training feasibility, and predictable pitfalls. While the work focuses on VR paradigms for neuropsychological assessment, many of the dimensions are also crucial for experimental research. Another similar framework has been proposed by an international working group of VR clinical outcomes research experts (VR-CORE). Their approach features three study phases (VR1-VR3) similar to the phase I-III model of pharmacological intervention studies $\left[9^{* *}\right]$. The focus is set on human-centered content development, feasibility, acceptability, tolerability, and clinical efficacy. Immersive VR also creates challenges for evaluation of user experience, and a model has been proposed to assess the interconnections between presence, engagement, immersion, flow, usability, skill, emotion, experience consequence, judgement, and technology adoption [46]. On the content side, VR software seeking high reliability should also include an ergonomic locomotion system, tutorials, in-game instructions and interactions similar to real-life [6**].

Based on the issues discussed in previous sections, we offer a list of concrete practical recommendations for designing and reporting of VR experiments to complement these general evaluation frameworks. Figure 1 presents a graphical summary of the guidelines.

\section{Guidelines for immersive VR research}

\section{Play VR games}

It is useful to learn what actually works in commercial practice [25], as game developers design their content so that a wide range of users would be able to enjoy the experiences comfortably and with high motivation for extended periods of time. With some luck, one might even find a ready-made game that can be easily used in an experiment.

\section{Beware of dual realities}

Consider the cognitive effects of "dual realities", as the knowledge that the virtual world is not actually real can have a confounding effect on the research phenomena of interest [27] and presence levels should be measured throughout the experiment [29*].

\section{Choose your setup}

Consider immersive VR as seated, stationary or free-roam experiences with new generation 6 degrees of freedom HMDs and high level of natural interaction [6**]. The particular choice of the VR paradigm and the level of immersion must ultimately depend on the research question [45 $\left.5^{\star \star}\right]$. 


\section{Design with human-centric principles}

Human-centric design principles must be kept in mind at all times, following VR industry best practices and avoiding cybersickness $\left[9^{* *}\right]$. An exception is for studies aiming to "break" some facet of virtual reality.

\section{Beautify}

Modern VR experiments should be up to participants' expectations about contemporary computer graphics seen in various games or movies, unless the goal of the study specifically states otherwise. Most modern game engines allow adding optimized shadows and anti-aliasing (smoothing of jagged edges), in fact they are the default settings. Complex and believable scenes increase ecological validity $\left[17^{*}\right]$.

\section{Gamify}

Add entertainment value or gamification aspects to increase the motivation of participants to wear the HMD for extended periods if needed. Top games in VR arcades can achieve an average session duration of around 30 minutes. Engaging content can help recruit a larger sample for statistical rigor $\left[16^{* *}\right]$.

\section{Pick your participants}

Keep in mind that individual differences in achieved levels of presence could be attributed to prior experience of VR $\left[1^{* *}\right]$. When recruiting participants, it is worth inquiring about the frequency of prior VR use.

\section{Test often and extensively}

Frequent pilot testing with participants is an unavoidable process of back-and-forth between different iterations of the study paradigm $\left[9^{* *}\right]$.

\section{Freeze updates}

Freeze software and hardware updates during the data gathering period of the main experiments if possible, as consumer VR middleware can introduce new unexpected features or break dependencies within the experimental software [7].

\section{Be real}

When interpreting study results, be realistic about the applicability of the VR study to real-world context $\left[1^{* *}, 16^{* *}, 26\right]$. 


\section{Foster replication}

The methods section of research papers should include information on play area size and exact physical setup. Source data, code and ideally the executable of the experience should also be available with the results to foster replication, review of data and procedures [16**].

\section{Guidelines for immersive VR research}

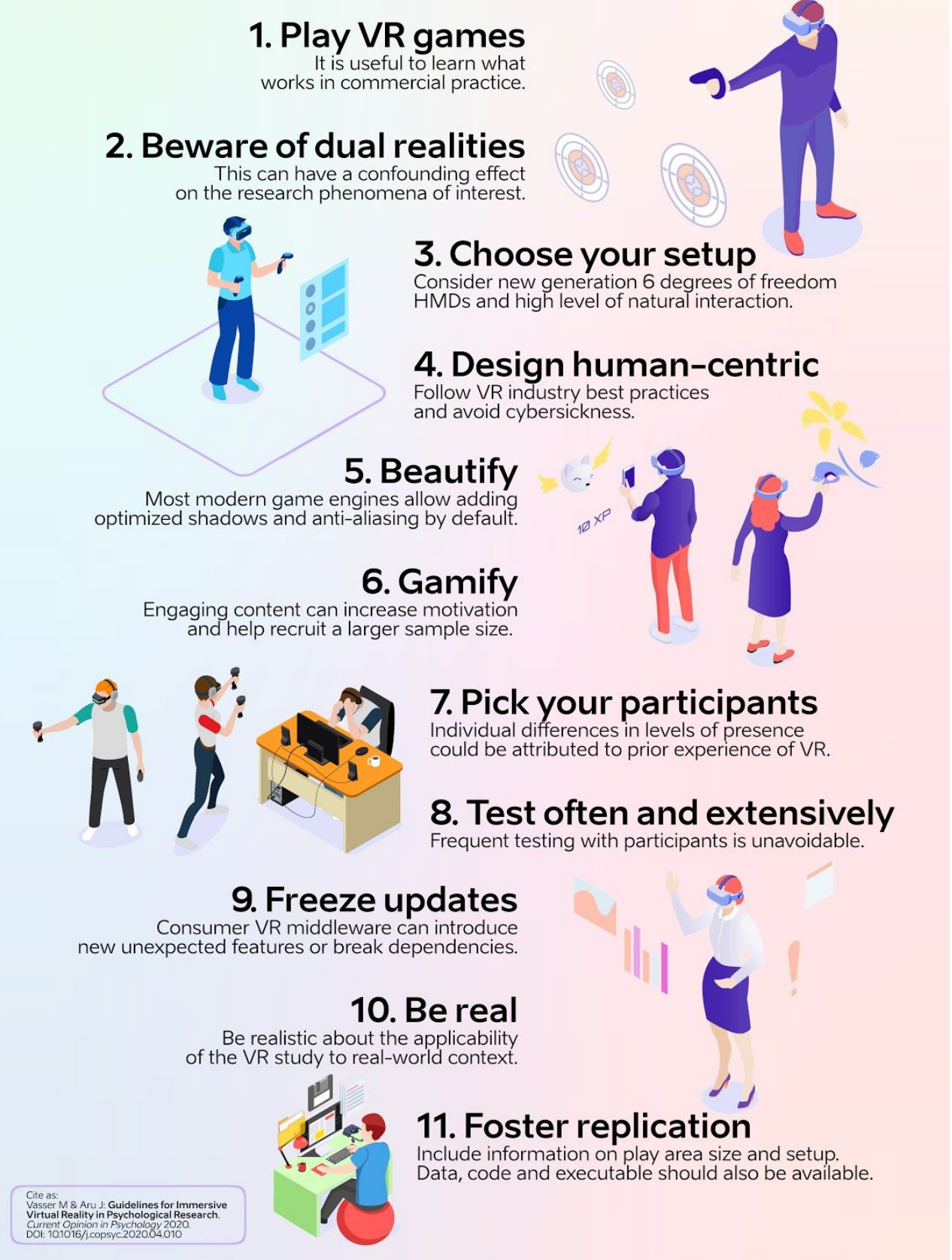

Figure 1. A graphical summary of the guidelines for immersive VR research 


\section{Conclusions}

While there is a plethora of experimental VR studies published, caution must be taken regarding the generalizability of these results to real-world interactions $\left[1^{* *}, 16^{* *}, 26\right]$. At the same time it is clear that immersive VR can provide novel insights about human behaviour and the workings of the brain given that the experiments are designed carefully. We hope that the proposed guidelines can steer the field from a "wild west" towards a scientific "gold rush".

\section{Acknowledgements}

We thank Michael Gaebler and Stephan Krohn for insightful comments on this manuscript. This work was supported by the European Union's Horizon 2020 Research and Innovation Programme under the Marie Skłodowska-Curie Grant Agreement No. 799411.

\section{Conflict of interest statement}

Nothing declared.

\section{CRediT authorship contribution statement}

Madis Vasser: Conceptualization, Writing - original draft.

Jaan Aru: Writing - review \& editing

\section{References and recommended reading}

Papers of particular interest, published within the period of review, have been highlighted as:

* of special interest

** of outstanding interest

1. ** Pan X, Hamilton AF de C: Why and how to use virtual reality to study human social interaction: The challenges of exploring a new research landscape. $\mathrm{Br} J$ Psychol 2018, 109:395-417.

Excellent review of state-of-the-art research that covers embodiment, the uncanny valley, simulation sickness, presence, ethics, and experimental VR design.

2. ** Miller LC, Shaikh SJ, Jeong DC, Wang L, Gillig TK, Godoy CG, Appleby PR, Corsbie-Massay CL, Marsella S, Christensen JL, et al.: Causal Inference in Generalizable Environments: Systematic Representative Design. Psychological Inquiry 2019, 30:173-202. 
The paper proposes Systematic Representative Design through virtual environments and other technologies in order to advance generalizability and robustness, cause-effect inference and precision of psychological research.

3. Peeters D: Virtual reality: A game-changing method for the language sciences. Psychon Bull Rev 2019, 26:894-900.

4. Rizzo A "Skip", Koenig ST: Is clinical virtual reality ready for primetime? Neuropsychology 2017, 31:877-899.

This article looks at past studies on VR therapies and declares that clinical VR is finally ready for primetime.

5. Slater M: Immersion and the illusion of presence in virtual reality. Br J Psychol 2018, 109:431-433.

6. ${ }^{* *}$ Kourtesis P, Collina S, Doumas LAA, MacPherson SE: Technological Competence Is a Pre-condition for Effective Implementation of Virtual Reality Head Mounted Displays in Human Neuroscience: A Technological Review and Meta-Analysis. Front Hum Neurosci 2019, 13:342.

A timely review of VR technology that emphasizes the importance of researchers' technological competency, meticulous methods and reports pertinent to software, hardware, and adverse symptoms.

7. Wiesing M, Fink GR, Weidner R: Accuracy and precision of stimulus timing and reaction times with Unreal Engine and SteamVR. PLOS ONE 2020, 15:e0231152.

8. * Yaremych HE, Persky S: Tracing physical behavior in virtual reality: A narrative review of applications to social psychology. Journal of Experimental Social Psychology 2019, 85:103845.

The review looks at different methods for tracing physical behavior in VR within the context of social psychology. 
9. ** Birckhead B, Khalil C, Liu X, Conovitz S, Rizzo A, Danovitch I, Bullock K, Spiegel B: Recommendations for Methodology of Virtual Reality Clinical Trials in Health Care by an International Working Group: Iterative Study. JMIR Ment Health 2019, 6:e11973.

This paper offers a three-step framework akin to pharmacotherapy for developing and evaluating VR treatments, focusing on content development, feasibility, acceptability, tolerability, and efficacy.

10. Sinesio F, Moneta E, Porcherot C, Abbà S, Dreyfuss L, Guillamet K, Bruyninckx S, Laporte C, Henneberg S, McEwan JA: Do immersive techniques help to capture consumer reality? Food Quality and Preference 2019, 77:123-134.

11. ** Yildirim Ç, Bostan B, Berkman Mi: Impact of different immersive techniques on the perceived sense of presence measured via subjective scales. Entertainment Computing 2019, 31:100308.

This research shows that many commonly used self-report presence questionnaires are not sensitive enough to differentiate between modern immersive techniques.

12. Borrego A, Latorre J, Alcañiz M, Llorens R: Comparison of Oculus Rift and HTC Vive: Feasibility for Virtual Reality-Based Exploration, Navigation, Exergaming, and Rehabilitation. Games for Health Journal 2018, 7:151-156.

13. Saredakis D, Szpak A, Birckhead B, Keage HAD, Rizzo A, Loetscher T: Factors Associated With Virtual Reality Sickness in Head-Mounted Displays: A Systematic Review and Meta-Analysis. Front Hum Neurosci 2020, 14:96.

14. Niehorster DC, Li L, Lappe M: The Accuracy and Precision of Position and Orientation Tracking in the HTC Vive Virtual Reality System for Scientific Research. i-Perception 2017, 8:204166951770820. 
15. de la Rosa S, Breidt M: Virtual reality: A new track in psychological research. $\mathrm{Br} J$ Psychol 2018, 109:427-430.

16. ** Lanier M, Waddell TF, Elson M, Tamul DJ, Ivory JD, Przybylski A: Virtual reality check: Statistical power, reported results, and the validity of research on the psychology of virtual reality and immersive environments. Computers in Human Behavior 2019, 100:70-78.

This meta-analysis looks at publication bias, questionable research practices, prevalence of errors and data availability of 61 different articles employing immersive VR research.

17. * Ramirez EJ: Ecological and ethical issues in virtual reality research: A call for increased scrutiny. Philosophical Psychology 2019, 32:211-233.

An in-depth study considering different ways to enhance or degrade context-realism and perspectival-fidelity of VR studies for ecological validity and coining the term "Virtually-real" experiences.

18. * Lindner P, Miloff A, Hamilton W, Reuterskiöld L, Andersson G, Powers MB, Carlbring $P$ : Creating state of the art, next-generation Virtual Reality exposure therapies for anxiety disorders using consumer hardware platforms: design considerations and future directions. Cognitive Behaviour Therapy 2017, 46:404-420.

This literature review details the current state of VR technology and highlights considerations about platform choice, software design and gamification.

19. ** Slater M, Gonzalez-Liencres C, Haggard P, Vinkers C, Gregory-Clarke R, Jelley S, Watson Z, Breen G, Schwarz R, Steptoe W, et al.: The Ethics of Realism in Virtual and Augmented Reality. Front virtual real 2020, 1:1.

This article considers the possible ethical implications of forthcoming "superrealistic" experiences and calls for creating a virtual and augmented reality code of conduct. 
20. de Gelder B, Kätsyri J, de Borst AW: Virtual reality and the new psychophysics. Br J Psychol 2018, 109:421-426.

21. * Riva G, Wiederhold BK, Mantovani F: Neuroscience of Virtual Reality: From Virtual Exposure to Embodied Medicine. Cyberpsychology, Behavior, and Social Networking 2019, 22:82-96.

This meta-review argues that VR treatments are effective due to embodied simulations, leading to the possibility of embodied medicine.

22. * Roberts AC, Yeap YW, Seah HS, Chan E, Soh C-K, Christopoulos GI: Assessing the suitability of virtual reality for psychological testing. Psychological Assessment 2019, 31:318-328.

This study finds that when the physical and virtual worlds are visually identical, psychological experiments yield comparable results, thus lowering the worry that VR itself might induce some bias.

23. Zimmer P, Wu CC, Domes G: Same same but different? Replicating the real surroundings in a virtual trier social stress test (TSST-VR) does not enhance presence or the psychophysiological stress response. Physiology \& Behavior 2019, 212:112690.

24. Monti A, Aglioti SM: Flesh and bone digital sociality: On how humans may go virtual. Br J Psychol 2018, 109:418-420.

25. Michela A, van Rooij MMJW, Klumpers F, van Peer JM, Roelofs K, Granic I: Reducing the Noise of Reality. Psychological Inquiry 2019, 30:203-210.

26. Kulik A: Virtually the ultimate research lab. Br J Psychol 2018, 109:434-436.

27. Pan X, Hamilton AF de C: Understanding dual realities and more in VR. Br J Psychol 2018, 109:437-441. 
28. Brogni A, Slater M, Steed A: More breaks less presence. In Presence 2003: The 6th Annual International Workshop on Presence. . Oct 6:1-4.

29. * Mai C, Thiem N, Hussmann H: DrawingPresence: A Method for Assessing Temporal Fluctuations of Presence Status in a VR Experience. Proc ACM Interact Mob Wearable Ubiquitous Technol 2019, 3:1-21.

The paper presents a novel and simple way of measuring the temporal aspects of presence after the experiment.

30. Pillai JS, Schmidt C, Richir S: Achieving Presence through Evoked Reality. Front Psychol 2013, 4.

This paper argues for concepts like evoked reality and evoked presence, constructing a three-pole model of reality that is useful for conceptualizing and navigating the phenomenon of virtual reality.

31. Harth J, Hofmann A, Karst M, Kempf D, Ostertag A, Przemus I, Schaefermeyer B: Different Types of Users, Different Types of Immersion: A User Study of Interaction Design and Immersion in Consumer Virtual Reality. IEEE Consumer Electron Mag 2018, 7:36-43.

32. Johns C, Nunez D, Daya M, Sellars D, Casanueva J, Blake E: The interaction between individuals' immersive tendencies and the sensation of presence in a virtual environment. In Virtual Environments 2000. . Springer, Vienna; 2000:65-74.

33. Peck TC, Sockol LE, Hancock SM: Mind the Gap: The Underrepresentation of Female Participants and Authors in Virtual Reality Research. IEEE Trans Visual Comput Graphics 2020, 26:1945-1954.

34. ** Harris DJ, Buckingham G, Wilson MR, Vine SJ: Virtually the same? How impaired sensory information in virtual reality may disrupt vision for action. Exp Brain Res 2019, 237:2761-2766.

The authors discuss how fewer binocular depth cues and conflicting depth information inherent in modern VR displays may create a fundamental disparity between virtual and 
real-world actions.

35. Peeters D: A standardized set of 3-D objects for virtual reality research and applications. Behav Res 2018, 50:1047-1054.

36. Tromp J, Akbal M, Pohl L, Krohn S, Quinque EM, Klotzsche F, Villringer A, Gaebler M: OpenVirtualObjects: An open set of standardized and validated 3D household objects for virtual reality-based research, diagnostics, and therapy. In 2019 International Conference on Virtual Rehabilitation (ICVR). . IEEE; 2019:1-2.

37. Farmani $\mathrm{Y}$, Teather RJ: Evaluating discrete viewpoint control to reduce cybersickness in virtual reality. Virtual Reality 2020 , doi:10.1007/s10055-020-00425-x.

38. Dużmańska N, Strojny P, Strojny A: Can Simulator Sickness Be Avoided? A Review on Temporal Aspects of Simulator Sickness. Front Psychol 2018, 9:2132.

39. Vasser M, Vuillaume L, Cleeremans A, Aru J: Waving goodbye to contrast: self-generated hand movements attenuate visual sensitivity. Neuroscience of Consciousness 2019, 2019.

40. Lin J, Zhu Y, Kubricht J, Zhu S, Lu H: Visuomotor Adaptation and Sensory Recalibration in Reversed Hand Movement Task. In CogSci. . 2017.

41. Ye T, Qi S, Kubricht J, Zhu Y, Lu H, Zhu S-C: The Martian: Examining Human Physical Judgments across Virtual Gravity Fields. IEEE Trans Visual Comput Graphics 2017, 23:1399-1408.

42. Laak K-J, Vasser M, Uibopuu OJ, Aru J: Attention is withdrawn from the area of the visual field where the own hand is currently moving. Neurosci Conscious 2017, 2017.

43. Warren WH: Non-Euclidean navigation. J Exp Biol 2019, 222:jeb187971. 
44. Schettler A, Raja V, Anderson ML: The Embodiment of Objects: Review, Analysis, and Future Directions. Front Neurosci 2019, 13:1332.

45. ** Krohn S, Tromp J, Quinque E, Belger J, Klotzsche F, Rekers S, Chojecki P, Akbal M, McCall C, Villringer A, et al.: Multidimensional Evaluation of Virtual Reality Paradigms in Clinical Neuropsychology: Application of the VR-Check Framework. J Med Internet Res 2020, doi:10.2196/16724.

This article proposes a thorough 10-step evaluation framework for VR applications in clinical neuropsychology that is also useful for assessing experimental VR paradigms.

46. Tcha-Tokey K, Christmann O, Loup-Escande E, Loup G, Richir S: Towards a Model of User Experience in Immersive Virtual Environments. Advances in Human-Computer Interaction 2018, 2018:1-10. 